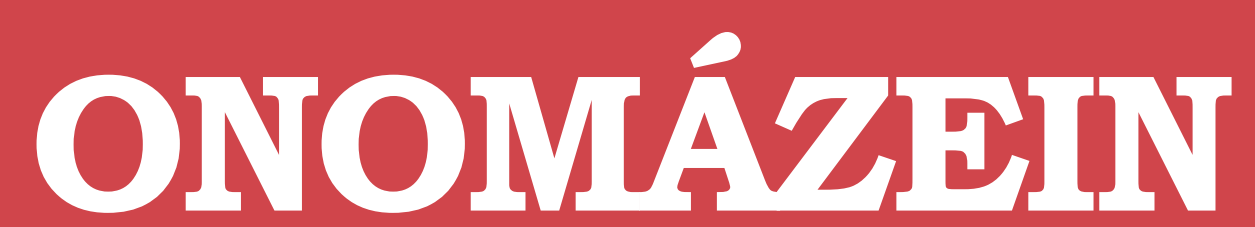

Revista semestral de lingüística, filología y traducción
PONTIFICIA UNIVERSIDAD CATÓLICA DE CHILE CATOLICA DE CHILE
FACULTAD DE LETRAS

\title{
Semantic density: A translation device for revealing complexity of knowledge practices in discourse, part 1-wording
}

\author{
Karl Maton \\ The University of Sydney \\ Australia
}

\author{
Y. J. Doran \\ The University of Sydney \\ Australia
}

Número especial
ONOMÁZEIN Número especial LSF y TCL sobre educación y conocimiento (2017): 46-76 DOI: 10.7764/onomazein.sfl.03

\section{(c) $\bigodot_{\mathrm{BY}} \bigodot_{\mathrm{ND}}$}

Karl Maton: LCT Centre for Knowledge-Building, The University of Sydney, Australia.

| Correo electrónico: karl.maton@sydney.edu.au

Y. J. Doran: Department of Linguistics, The University of Sydney, Australia.

| Correo electrónico: yaegan.doran@sydney.edu.au

Fecha de recepción: 2 de octubre de 2015

Fecha de aceptación: 29 de enero de 2016 


\section{Abstract}

In education research 'complexity' is often viewed cognitively as a mental attribute and so the complexity of knowledge practices themselves remains underexplored. Legitimation Code Theory conceptualizes such complexity as 'semantic density', which describes how meanings are condensed and interrelated within knowledge practices. This concept is becoming widely enacted in education as a means of identifying and teaching highly-valued practices. As yet, how 'semantic density' could be enacted to analyse the discourse of actors remains uncertain. This paper is the first of two articles that introduce a means of enacting the concept in analysis of English discourse. Together they offer a 'translation device' that explores discourse for signs of the complexity of the knowledge being expressed. This first paper introduces tools for exploring how the wording used by actors realizes different strengths of 'epistemic-semantic density', where meanings are empirical descriptions or formal definitions. It provides typologies for identifying different kinds of wording and describes how these types manifest different degrees of complexity. Two contrasting examples, from a secondary school History classroom and a scientific research article, are analysed to illustrate the insights into complexity offered by these tools. In the second paper we build on these ideas with tools for analysing how words are combined to generate different degrees of increasing complexity, to enable a fuller understanding of knowledge-building.

Keywords: Legitimation Code Theory; semantic density; translation device; language of description; complexity; knowledge-building. 


\section{Introduction}

Complex, sophisticated, profound, deep..., such terms are often used to describe highly-valued knowledge practices in education but often obscure more than they reveal. Scratch the surface and one finds they are black boxes that indicate something widely held to be significant but which proves persistently difficult to define. Ironically, our understanding of the complexity of knowledge practices is anything but complex. In this paper and its sequel, 'Condensation' (henceforth 'Paper 2'), we introduce semantic density from Legitimation Code Theory to conceptualize complexity in knowledge practices and outline a multipart translation device for enacting this concept in analysis of English discourse. We begin by explaining, first, why this concept is needed and, second, how this focus on analysing discourse arose in collaborative research with systemic functional linguistics (henceforth 'SFL')'.

\subsection{Semantic density}

In academic discourse words such as 'complex', 'abstract' and 'sophisticated' are often employed but rarely defined, as if their meanings are transparent. Yet, they typically elide a shifting and poorly defined set of issues, including relations between concepts and examples, between theory and practice, and among ideas. In education research the issue of complexity is the ostensible subject of a voluminous body of work. However, this research overwhelmingly focuses on the mental aspects of learning by exploring the cognitive demands of tasks. Reflecting the 'subjectivist doxa' (Maton, 2014b) that dominates education research, 'knowledge' is typically viewed as comprising states of mind or dispositions to act. While valuably revealing characteristics of what is more accurately termed 'knowing', this psychological focus provides less insight into 'knowledge' itself. Taking classroom studies as an example, research mostly focuses on the cognitive complexity of learning tasks (such as ranging from short-term memorization to critical evaluation) and neglects the forms taken by the knowledge practices being learned. The notion that 'knowledge' can be an object for study in its own right, one that possesses properties and powers with real effects, has been largely obscured².

1 We are grateful to Talia Gill for contributing to earlier versions of the tools and to Eszter Szenes for feedback on a pilot wording tool. This work forms part of the PEAK Project, supported by the Australian Research Council (Discovery Project, DP130100481).

2 The subjectivist doxa takes different forms across the segments of education research, negating detailed engagement here; see, for example, Georgiou et al. (2014) on physics education research, where complexity is a significant issue. 
The tradition of code theory begun by Basil Bernstein offers a means of seeing knowledge practices as an object of study. However, even here the issue of complexity has received relatively little attention. Bernstein's concepts (2000) only indirectly allude to the issue. Studies of science curriculum have drawn on his concept of 'classification' to describe strengths of boundaries among teaching units and between theory and practice, to which scales of cognitive difficulty and 'complexity of scientific knowledge' (a typology of facts - simple concepts - complex concepts - unifying themes/theories) are then added to describe 'conceptual demand' on learners (e.g. Calado et al., 2013; Morais et al., 2004). However, the themes underlying these diverse measures of distance from empirical contexts and of relations among ideas have not themselves been explicitly conceptualized within the framework. They offer means for measuring issues that have yet to be fully theorized.

Legitimation Code Theory (LCT) extends and integrates Bernstein's approach to conceptualize context-dependence as semantic gravity and relations among meanings as semantic density. These concepts were first introduced in Maton $(2009,2011)$ and more fully elaborated in Maton (2013, 2014a, 2014b, 2016a). In these two papers we focus on 'semantic density', which conceptualizes complexity in terms of the condensation of meanings within practices (symbols, concepts, expressions, gestures, actions, clothing, etc.). The strength of semantic density can vary along a continuum. The stronger the semantic density (SD+), the more meanings are condensed within practices; the weaker the semantic density (SD-), the fewer meanings are condensed. Put another way, semantic density explores the relationality of meanings: the more meanings are related, the stronger the semantic density. The notion of relationality is illustrated by Figure 1 . If we state 'there is a Gwiffly' (a previously meaningless word), we have established a solitary node of meaning (number 1 in Figure 1 ). If we then describe two kinds of Gwiffly as 'A' and 'B' (number 2), we have established relations between 'Gwiffly' and two further terms. If we then describe their characteristics, such as 'Gwiffly $A$ is red and Gwiffly B is blue', we are augmenting those terms by relating them to more meanings (number 3 ). This begins to create a 'constellation' of meanings (Maton, 2014b: 148-170), a process that can be continued indefinitely (number 4). The more relations established with other meanings, the stronger the semantic density.

\section{FIGURE 1}

Constellating a Gwiffly
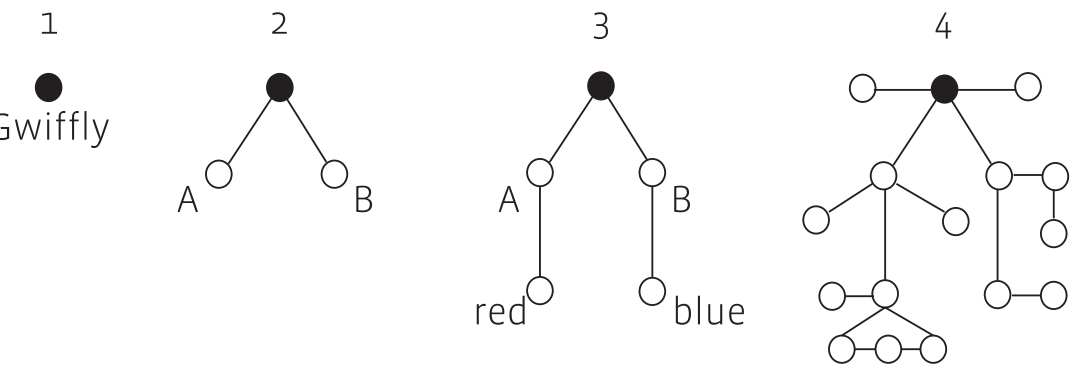
This process of adding meanings to a symbol or practice is termed condensation. As we shall illustrate in Paper 2, condensation takes many forms. It may also involve different kinds of meanings. Our concern in these papers is with epistemic-semantic density based on the epistemological condensation of formal definitions and empirical descriptions ${ }^{3}$. For example, the paper you are now reading involves epistemological condensation of formal definitions and empirical illustrations into the term 'epistemic-semantic density'. This form can be distinguished from, among other kinds, axiological-semantic density based on axiological condensation of affective, aesthetic, ethical, political or moral stances (Maton, 2014: 153-170). For example, Martin et al. (2010) explore the ways in which political meanings are condensed into terms such as 'nationalism' and 'colonialism' in secondary school History classrooms. Describing our concepts as 'beautiful' would offer another example of axiological condensation.

The concept of 'semantic density' is being widely enacted in education research and practice to reveal and teach one facet of the 'rules of the game' (e.g. Maton et al., 2016b; Doran, this issue $)^{4}$. Studies suggest that combinations of strengthening and weakening semantic density are rewarded in student work and help to build knowledge in classroom practices (Blackie, 2014; Clarence, 2014; Macnaught et al., 2013; Maton, 2014a; Matruglio et al., 2013). Moreover, the concept is being enacted to explore other social fields, including public understanding of climate change (Glenn, 2015). As this suggests, 'semantic density' is highly flexible. In education, for example, the concept can be enacted to explore diverse practices (research, curriculum, pedagogy, etc.) at all levels (from education system to a single word) and across all disciplines. Unsurprisingly, the forms taken by semantic density vary across these practices and contexts. Thus, a key task is to establish its empirical realizations within a particular object of study and to make this explicit, often in the form of a 'translation device' that systematically relates the concept to data. In these papers we outline a multipart translation device for enacting 'epistemic-semantic density' in analyses of English discourse.

\subsection{A translation device for discourse}

The origins of this translation device are the interdisciplinary DISKS (2009-11) and PEAK (2012-) projects in which LCT and SFL have been enacted together to explore classroom practice (Martin \& Maton, this issue). In the DISKS project, LCT analysis showed how shifts in the strengths of semantic gravity and semantic density are crucial to knowledge-building (Maton, 2013), and SFL analyses revealed the complex language choices associated with those shifts (Martin,

3 Thus, more accurate (but less reader-friendly) titles for our two papers would be: 'Epistemic-semantic density' and 'Epistemological condensation'.

4 For papers, see http://www.legitimationcodetheory.com 
2013a). Enacting the two frameworks in close analyses of the same data encouraged theoretical innovations (e.g. Martin, 2013b; Martin, this issue). As Maton et al. (2016a: 108) describe, 'what scholars using the other framework want to know can encourage the development of concepts that make new things visible'. After DISKS, one thing J. R. Martin wanted to know was how the semantic density of knowledge practices could be seen in the written and spoken discourse of secondary school History and science classrooms. This helped encourage the development of a translation device that is unusual both for its breadth (to which we return in section 2.1) and for its concern with discourse, which we shall discuss here.

While the concepts of LCT can be enacted to explore phenomena as diverse as music notation, body movement and architecture, thus far most studies drawing on the framework have focused on educational practices. Accordingly, translation devices for enacting concepts typically include such indicators as:

- 'Student is describing a physical principle, law, theory or concept in a general enough way that it means something without reference to a specific concept' (Georgiou et al., 2014: 259)-indicator of relatively weak semantic gravity of student work in university physics; and

- 'Content knowledge is emphasized as determining the form of legitimate educational knowledge' (Chen et al., 2011: 134)—indicator of relatively strong epistemic relations of curriculum knowledge in interviews with Chinese students.

These examples describe how specific organizing principles (semantic gravity and epistemic relations) are expressed in specific data (student work and interviews) about specific objects of study (undergraduate physics and Chinese students' experiences). This is the typical form taken by indicators enacting LCT. Their purpose is to translate a concept into descriptions of practices recognizable within a determinate problem-situation. Indicators of this kind for, say, relatively strong epistemic-semantic density in classroom practices could include 'stating an overarching law or unifying theory that combines a number of concepts' or 'conducting a practical experiment representing multiple variables'. Such descriptors of practices would offer valuable means for enacting the concept in classroom research. However, they are not our focus here. Instead we are concerned with how epistemic-semantic density may be realized in discourse. This is to highlight a difference in the phenomenon to which the concept is being translated. The kinds of educational practices typically explored by LCT research may be expressed through discourse (among other things), but thus far the discourse itself has not been the principal focus (and rightly so, given the concerns of that research). Thus the translation device we outline is not intended to displace the kinds of indicators illustrated above but rather to complement their focus on practices: it offers a means of additionally analysing the discourse through which those practices may be (at least partly) expressed. 


\section{FIGURE 2}

Concept, translation device and phenomenon

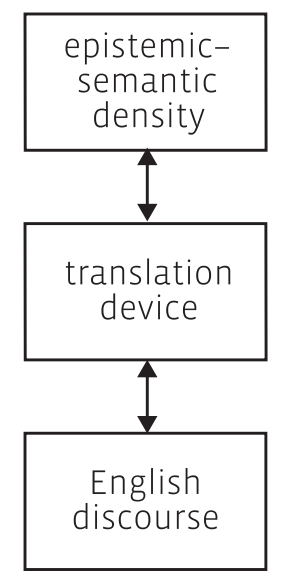

However, discussing discourse is dangerous. Scholars often suffer from a substantive bias that views any concept relating to a phenomenon as a model of that phenomenon. A translation device involving discourse may thus be misrepresented as 'linguistics', 'sociolinguistics', or 'a sociology of language'. This is to fundamentally misunderstand its purpose and nature. As illustrated by Figure 2, the translation device is a means for translating between the higher-order concept of epistemic-semantic density and English discourse. To do so the device offers a series of typological categories at different levels of discourse (wording, word-grouping, clausing, sequencing) that represent different strengths of epistemic-semantic density and epistemological condensation (see section 2.1). However, these typologies are not linguistic concepts, do not derive from linguistics, and do not represent models of language. They are sociological concepts for revealing an attribute of knowledges. The categories provide a means of 'reading' English discourse for signs of epistemic-semantic density; the basis of categories always remains strengths of epistemic-semantic density rather than attributes of language. Some characteristics of categories may resonate with notions from linguistics; given the phenomenon, it is hard to imagine otherwise. However, such characteristics are relevant here only for revealing strengths of epistemic-semantic density. For example, when discussing the term 'lipopolysaccharide' (section 3.2), we highlight two signs of relatively strong epistemic-semantic density: its location within a specialized domain of social practice (the academic discipline of biology); and its evocation of complex constellations of knowledge through multiple distinct parts that each possess a technical meaning in biology. As we show with the contrasting example of 'supercalifragilisticexpialidocious', the characteristic of appearing to possess many parts is relevant only

5 Thus, following Bernstein, we refer to 'discourse' rather than 'language'; for example, 'horizontal discourse' and 'vertical discourse' (2000) describe features of knowledge rather than language. 
if it reflects epistemic-semantic density. Moreover, all other formal linguistic features of these terms are irrelevant here: our only concern is epistemic-semantic density.

Put another way: knowledge is expressed in a number of ways, one of which is through discourse; the translation device explores how that expression in discourse might reveal one characteristic of the knowledge being expressed. Thus, the tools we outline are neither exhaustive of epistemic-semantic density (which may be realized in different ways through body movement, images, architecture, etc.) nor an LCT model of discourse (which would need to additionally capture axiological-semantic density, semantic gravity, epistemic relations, social relations, and other constitutive principles of legitimation codes). They refer to only one form (epistemic-) of one organizing principle (semantic density) of practice as realized in one phenomenon (English discourse).

We labour this point because these two papers are unusual for a sociological framework. They are primarily definitional, involve a high number of technical terms, and introduce a means of enacting a concept within fine-grained analysis of discourse. This may be viewed by linguists as a hostile incursion onto their terrain and by sociologists as a failure to remain sufficiently sociological. However, Halliday stated that a 'discipline is really defined by the questions you are asking' (in Martin, 2013C: 128-9) and Bernstein argued that 'what makes something sociological has nothing to do with the phenomena, but everything to do with the conceptual system to which the phenomena is referred' (1973: 7). Here we are asking sociological questions about discourse using a concept embedded within the sociological framework of LCT and, more widely, code theory ${ }^{6}$. The resulting tools enable research to address such sociological questions as who gets to define what is possible, for whom, when, where and how. Nonetheless, we hope the translation device enables other researchers to ask their own questions, whatever their discipline, for the tools are intended to help solve problems. We shall begin by explaining the nature of the translation device and outlining how the tools will be discussed in these two papers.

\section{The translation device}

\subsection{A mediating language}

Earlier above (section 1.2) we stated that the translation device is unusual for its breadth. Extending Bernstein's model (2000) of 'languages of description', Maton (2016b) distinguishes between two kinds of translation device:

6 For example, the influence of Bernstein's model of 'discourses' and 'knowledge structures' (2000) on the sequencing tool (Paper 2) will be obvious. 
- external languages that relate concepts to data within the problem-situation of a specific study; and

- mediating languages that relate concepts to data but aim at embracing all empirical forms of a phenomenon (such as all English discourse or all images).

External languages are bespoke translation devices between a specific concept and specific data about a specific object of study (see Maton \& Chen, 2016). Such devices are often presented as a table outlining: settings of a concept (e.g. stronger/weaker epistemic relations); indicators of those settings in the study (such as those discussed earlier above); and empirical examples matching those indicators. In contrast, a mediating language embraces a diverse range of empirical realizations of a concept across a broader object of study. Typically, this additionally requires intermediate concepts, such as typologies, to translate between the higher-level concept and the phenomena.

As discussed above, our translation device originated from interest in how epistemic-semantic density is realized in classroom discourse of secondary school History and science. Such a specific focus would normally engender an external language tailored to this object of study. However, similar queries from scholars researching other objects of study broadened our focus to embrace English discourse as a whole, necessitating a mediating language that includes intermediate concepts. Specifically, the translation device comprises four sets of typologies for analysing:

- strengths of epistemic-semantic density (henceforth ESD) at the level of words (wording and word-grouping tools); and

- degrees of epistemological condensation or the strengthening of ESD by combining words into short passages (clausing tool) and by combining short passages into longer sequences of discourse (sequencing tool).

Put simply, these tools explore the complexity of meaning expressed by words and how that complexity is increased through their combination with other words. In this paper we outline how to analyse ESD in discourse: wording and word-grouping tools. The rest of this section introduces the method whereby these typologies were created, key attributes of ESD in discourse, and limits of the tools. Section 3 outlines the wording tool and section 4 describes the word-grouping tool. Section 5 illustrates both tools through analyses of excerpts from a secondary school History lesson and a scientific research article. In Paper 2, we outline the clausing and sequencing tools for exploring epistemological condensation in English discourse and build on the current paper by analysing the same examples to show how the translation device works as a whole. Thus, while the tools (with the exception of word-grouping) can be enacted separately to explore different facets of discourse, the two papers together show how combining tools generates additional insights. 


\subsection{Creating the wording tool}

ESD traces a continuum of strengths. Allocating specific instances of discourse to positions along the continuum requires identifying categories of wording that equate to different strengths. To achieve this end we undertook a process of recursive division of the continuum, as illustrated in Figure 3. We began by looking for the most salient and robust basis for a division of the data into stronger and weaker ESD. This eventually generated two categories ('Types' in Figure 3). Second, taking each type separately we looked for the most salient and robust basis of division of that type into categories of stronger and weaker ESD within that type. This generated four categories ('Subtypes' in Figure 3). Third, we repeated this division within each of the four subtypes, to generate eight categories ('Sub-subtypes').

\section{FIGURE 3}

Recursive division of epistemic-semantic density from a continuum into categories

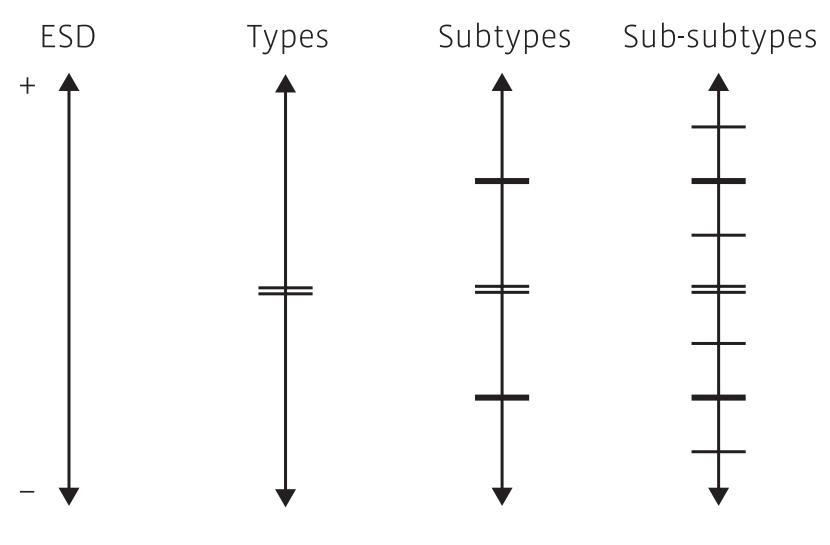

The data comprised classroom discourse from the DISKS project, academic writing from across the sciences and humanities, and everyday conversation. Put simply, after each stage of development we repeatedly crash-tested the tool by searching for examples that failed to fit the categories, casting our net as widely as possible, from science to pop songs, from research to slang. We repeated this process until new data no longer reshaped the typologies, while limiting the number of resultant categories to a minimum?. The aim was to exhaust possible data without, we hope, the result exhausting researchers-a balance between the degree of delicacy offered (for the process of division is potentially endless) and the practical need for an appliable instrument. This is, of course, a matter of judgement and does not foreclose de-

7 Space precludes outlining the less linear process truncated by this description; over three years numerous versions were created, refined, publicly discussed, reworked, scrapped, etc. We are grateful to Sydney Roundtable participants for their engagement with several versions. 
veloping further sub-categories if required to explain a problem-situation. (Indeed, these two papers are replete with suggestive possibilities for further levels of delicacy).

Conversely, while more concepts may be developed in future, one may not need all the concepts introduced here. The translation device is a toolbox; which tools are required depends on the problem. If complexity (ESD) is an issue when analysing discourse, then the wording tool (and possibly word-grouping) may be useful; if building complexity (epistemological condensation) is a concern, then the clausing and/or sequencing tools may be relevant. Moreover, within each tool one only needs as much delicacy as the problem demands. Each tool typically offers three levels of types, but one need not use them all. One can stop at any level. However, one cannot start at any level. Subtypes distinguish within types rather than offering an alternative typology. (For example, in section 3 subtypes of 'technical' words are distinguished along different lines to subtypes of 'everyday' words). One must thus identify types before distinguishing their subtypes. This hierarchy is shown by the horizontal lines of Figure 3 and tables setting out each tool: double lines divide types, heavy single lines divide their subtypes, and lighter single lines divide their sub-subtypes. To facilitate this understanding we also introduce each tool separately and, within each tool, first types and then finer distinctions within each type.

\subsection{The nature of the device and attributes of ESD}

Though embracing the broad phenomenon of English discourse, the translation device has limits to both its specificity and its generality. In terms of specificity, enacting the device to explore determinate forms of English discourse may require external languages for translating its concepts into their particularities. We shall outline typological categories (in tables) of different strengths of ESD and provide descriptions (in the main text) of indicators, typical characteristics and empirical examples of each category. However, space precludes capturing all possible characteristics and empirical forms taken by each category. The characteristics we highlight are commonly-found features they are 'rules of thumb' for identifying categories rather than definitive and universal. Thus our frequent use of 'tends', 'likely', 'often', 'usually' and 'typically'. Each tool offers a table of tendencies intended to assist empirical research by suggesting where words might be placed along the continuum of ESD. Similarly, the examples illustrating categories are not exhaustive. Enacting the device, therefore, may require attending to the specificities of an object of study.

In terms of generality, this translation device is limited to English discourse. Exploring other languages may necessitate adapting these tools or creating new ones. Other semiotic resources_images, mathematical symbols, music notation, body movement, etc.-require their own translation devices. Nonetheless, the tools we outline offer suggestive principles 
for their development. Specifically, three features can be highlighted as associated with strengths of ESD and epistemological condensation: relationality, differentiation and resonance.

First, the more relations that a constituent of a constellation (such as a concept) enjoys with other constituents (such as other concepts), the stronger the ESD (see section 1). In effect, relationality describes the complexity of the constellation of which the unit under consideration is a constituent. Second, the more relations a meaning has with other meanings, typically the more differentiated those relations and so the more specific, fine-tuned and precise the referents of that meaning. Third, employing a constituent of a constellation tacitly resonates out to the rest of that constellation, whether mentioned or not. So, the more relations with other meanings, the more resonance outwards along those relations to meanings that are implied but not specified. All three attributes are illustrated by this example from biology: in comparison to 'vesicles', the term 'vesicles called lysosome' involves more relations, more differentiation and more resonance-it specifies that 'lysosome' is a type of 'vesicle', establishing two levels of a taxonomy and suggesting the possibility of other kinds of 'vesicle'. These three attributes, we suggest, are implicated in such widely-used but littledefined descriptions as 'profound', 'sophisticated' and 'deep': they highlight how the wording choices of actors are implicating complex constellations of meaning. They may also offer a sufficiently general starting point for developing further translation devices into other kinds of practices (see Paper 2, section 5).

\section{Wording}

The wording tool for establishing ESD is summarized in Table 1 and includes three levels of delicacy. The tool focuses on 'content words' - nouns, verbs, adjectives and adverbsrather than 'grammatical words' such as prepositions (e.g. 'in', 'at'), determiners (e.g. 'the', 'a') or conjunctions (e.g. 'and', 'or', 'however'), which proved less significant for analysis of ESD $^{8}$. In section 3.1 we define two principal categories of wording for ESD: technical and everyday. In section 3.2 we discuss conglomerate and compact forms of technicals; and in section 3.3 we discuss consolidated and common forms of everydays. In both sections we also introduce a third level of delicacy within each of these subtypes. In section 3.4 we address how this tool can also be used to categorize words (such as 'it') that act as 'proxies' for other words.

8 Prepositions and determiners are significant for epistemic-semantic gravity (see future publications) and conjunctions are significant for epistemological condensation in sequencing (see Paper 2). 


\section{TABLE 1}

Wording tool for epistemic-semantic density

\begin{tabular}{|c|c|c|c|}
\hline ESD & Type & Subtype & Sub-subtype \\
\hline \multirow{4}{*}{$\stackrel{+}{\uparrow}$} & \multirow{4}{*}{ technical } & \multirow{2}{*}{ conglomerate } & -properties \\
\hline & & & -elements \\
\hline & & \multirow{2}{*}{ compact } & -properties \\
\hline & & & -elements \\
\hline \multirow{4}{*}{$\downarrow$} & \multirow{4}{*}{ everyday } & \multirow{2}{*}{ consolidated } & specialist \\
\hline & & & generalist \\
\hline & & \multirow{2}{*}{ common } & nuanced \\
\hline & & & plain \\
\hline
\end{tabular}

\subsection{Wording types}

The first level of delicacy explores whether a word depends for its meaning on being located within a specialized domain of social practice ${ }^{9}$. This distinguishes between:

- technical words (ESD+) whose meanings are given by their location within a specialized domain of social practice; and

- everyday words (ESD-) whose meanings are given not by their location in such specialized domains but rather through their usage in commonplace practices and contexts.

This emphasizes the nature of the constellations of epistemic meanings within which a word is located. The epistemological constellations developed by specialized social fields, such as academic disciplines, often tend towards greater complexity, depth and stability, while those generated in everyday practices often tend towards being more transitory, mobile, shifting and flexible in their relations. Thus, where the meaning of a word is dependent on a specialized field it is likely to exhibit stronger ESD ${ }^{10}$.

9 The term 'specialized' describes social fields whose relatively high autonomy have enabled a distinctive set of social practices to emerge and so embraces all 'uncommonsense' fields, including academic discourse, professions, crafts, sports, etc. (It is far broader than the SFL denotation of trades, arts and crafts).

10 This is not suggesting everyday words are simpler in every way. To reiterate, our concern is solely with epistemic-semantic density. The constellations of axiological meanings generated in everyday contexts may be more complex than those of specialized fields and so everyday words may have stronger axiological-semantic density than technical words. So they may be more complex in another way. Moreover, ESD strength is always a matter for empirical research (see section 6). 
For example, as an everyday word the term 'gold' commonly denotes a bright yellow, shiny and malleable metal used in coinage, jewellery, dentistry, and electronics. In everyday contexts, its epistemic meanings thus involve a relatively limited number of relations to other epistemic meanings. However, as a technical word within the academic discipline of Chemistry, the term is related to an atomic number, atomic weight, electron configuration, and much more. Many of these meanings involve relations to other meanings as part of compositional structures, taxonomies and explanatory processes; for example, its atomic number represents the number of protons found in the nucleus of an atom, identifies it as a chemical element, and situates it within the periodic table. Thus, 'gold' in Chemistry is located within an extremely complex epistemological constellation that imbues the term with a greater range of epistemic meanings. Similarly, the word 'equestrian' in the discipline of History refers to the lowest aristocratic class in the Roman military and thereby resonates across a taxonomy and hierarchy of classes and relates to a delimited period in history, specific political relations, etc. It is thus a technica/ word distinct from its everyday usage relating to horses.

As these examples illustrate, strength of ESD is not intrinsic to a word but rather depends on the epistemological constellation of meanings to which the word is related. The same word may be used as both a technical and an everyday word; words born as technicals may be used in everyday contexts shorn of many epistemic meanings; and words originating as everydays may become incorporated into a specialized epistemological constellation and thereby 'technicalized'. Accordingly, wherever there is potential for such double usage, our examples shall include sufficient context to indicate whether the word is technical or everyday.

In terms of typical characteristics, technicals are usually nouns, often long words (see section 3.2), and may be names of people (such as 'Plato' or 'Beethoven' where referring to a corpus of work in philosophy or music) or places (such as 'Pompeii' when referring in History to specific events, societal relations, time periods, etc.). Examples (underlined) include:

- 'The phagocyte moves into the area of invasion and then attaches to the microorganism'.

- 'One of the things we know about Pompeii and houses is that they don't have windows'.

The meanings of technicals are often assumed within their specialized domain, unless located in pedagogic settings or when first introduced in research articles, where their technical nature may be highlighted, such as by quote marks or (the convention in LCT) italics. The capacity of technicals to resonate across complex epistemological constellations is a significant contributor to a text feeling of potential significance to specialist readers and 'dense' to non-specialist readers.

Everydays often tend to be shorter than technicals and include all word classes (nouns, verbs, adjectives etc.), such as: 
- 'The phagocyte moves into the area of invasion and then attaches to the microorganism.'

- 'One of the things we know about Pompeii and houses is that they don't have windows.'

As they are not embedded in constellations where epistemic meanings and relations are explicitly defined and relatively stable, everydays can be related to a very high number of other words without losing their meaning, allowing more fluid and shifting constellations. This may involve less differentiation, and thus epistemic precision, but greater flexibility.

\subsection{Subtypes of technicals}

As shown in Table 1, technicals can be distinguished into cong/omerates and compacts; and everydays can be distinguished into consolidateds and commons. In each case, the longer "con-" represents stronger ESD than the shorter "com-".

The key distinction within technical words is between:

- conglomerate words that comprise multiple distinct parts that each possess a technical meaning; and

- compact words that comprise a single part with a technical meaning.

Compacts, as technical words, exhibit relatively strong ESD but not as strong as conglomerates. Examples (underlined) include:

- 'Lymph empties into the subclavian veins'

- 'The strophe is the first part in an ode'

Though comprising one meaningful part, they may have grammatical inflection or derivation (such as 'lymphatic' in comparison to 'lymph') but any inflection or derivation has no direct implication for ESD ${ }^{11}$.

Conglomerates exhibit stronger ESD because each of their parts involves relations to other meanings, such as specifying multiple levels of a taxonomy (a set of relations of meaning ordered by type and subtype). For example, in the quote 'We're going to look at lipopo-

11 They may have indirect implications through enabling words to occur in larger word-groupings (section 4), but do not change their ESD category. 
Iysaccharide now' the underlined term comprises: 'lipo' meaning 'lipid' (crudely 'fat'), 'poly' meaning 'multiple', and 'saccharide' denoting a carbohydrate. Each part resonates outwards along relations to other meanings: specifying 'lipo' implies there may be other types of polysaccharides (which there are, such as mucopolysaccharides); and specifying 'poly' implies there are other types of saccharide (which there are, such as oligosaccharides). The conglomerate nature of the word thus suggests a tacit taxonomy. Each part suggests it is one option among a range of possibles, implying relations to other meanings and thus potentially stronger ESD. Table 2 shows a portion of the classificatory taxonomy involving 'lipopolysaccharide'. As this highlights, one characteristic of conglomerates is often their greater differentiation than compacts; in this case, 'lipopolysaccharide' is more differentiated than 'polysaccharide', which in turn is more differentiated than 'saccharide'.

\section{TABLE 2}

Partial taxonomy of saccharides

\begin{tabular}{|l|l|l|}
\hline lipo & \multirow{2}{*}{ poly } & \\
\cline { 1 - 1 } muco & & \multirow{2}{*}{ saccharide } \\
\cline { 1 - 1 } exo & & \\
\cline { 1 - 1 } galacto & \multirow{2}{*}{ oligo } & \\
\cline { 1 - 1 } fructo & & \\
\cline { 1 - 1 } isomalto & & \\
\hline
\end{tabular}

For greater delicacy, one can further distinguish among conglomerates according to how many meaningfully distinct parts they comprise (C2 contains two meaningfully distinct parts, C3 contains three meaningfully distinct parts, etc). However, though often characterized by length, not all long words are conglomerates. First, they must be 'technicals', because subtypes are reached through types (see section 2.2); indeed, the subtypes are more accurately termed technical-conglomerates and technical-compacts. Second, the parts must be meaningful. Third, the meanings must indicate epistemological condensation. Thus, 'supercalifragilisticexpialidocious' is not a conglomerate because it is not a technical word (its meaning is not dependent on a specialized field of practice) and its parts (though said to have roots in specific words) are not epistemically meaningful ${ }^{12}$. Conversely, conglomerates need not be long. They may take the form of acronyms, such as 'HDL' ('High-Density Lipoprotein') or 'NATO' ('North Atlantic Treaty Organization'), into which epistemic meanings have been condensed.

12 'Even though the sound of it is something quite atrocious / If you say it loud enough, you'll always sound precocious' suggests the song devalorizes epistemological condensation but acknowledges the word could be misrecognized as a technical. 


\subsubsection{Finest level of technicals}

A third level of delicacy, detailed in Table 1 , distinguishes within conglomerates and compacts according to whether they denote:

- an item, entity or thing of some kind: (-elements); or

- an action (event, process, etc.) or quality of an item, entity or thing (-properties).

This distinction recurs within both conglomerates and compacts to delineate: conglomerateproperties and conglomerate-elements; and compact-properties and compact-elements.

Conglomerate-properties represent stronger ESD than conglomerate-elements and compact-properties represent stronger ESD than compact-elements because they not only denote an action or quality but also imply the elements involved in that action or possessing that quality. For example, 'grammar' is a compact-element in linguistics that refers to an item within language; 'grammaticalization' is a compact-property referring to a process. The latter is characterized by stronger ESD as it implicates the meanings associated with 'grammar' as well as other elements and the process itself. Similarly, 'phagocyte' is a conglomerate-element: it comprises 'phago-' (denoting 'devouring' or 'eating') and 'cyte' (denoting 'cell') and refers to an entity. 'Phagocytosis' is a conglomerate-property because it denotes a process (engulfment of a solid particle by a phagocyte). The latter exhibits stronger ESD than the former because the meanings associated with 'phagocyte' and the solid particle are enfolded within 'phagocytosis', which additionally involves meanings relating to the process whereby one engulfs the other.

The category of conglomerate-properties represents the strongest ESD in our wording tool, for the elements involved in the action or possessing the quality may each resonate out to complex sets of relations (such as taxonomies) and the action or quality itself may also be one of a range of types. Thus, the number of relations implicated in a single word can be considerable.

\subsection{Subtypes of everydays}

Typologies of knowledge often distinguish different forms of academic or uncommonsense knowledge but leave everyday or commonsense knowledge as a residual, undifferentiated category. In contrast, this wording tool describes a series of fine-grained categories for ESD in everyday wording. The first distinction is between:

- consolidated words that encode happenings or qualities as things; and 
- common words that leave happenings as happenings and qualities as qualities.

By 'happenings' we mean processes or events that are normally realized by verbs and by 'things' we mean elements or items (physical or intangible) that are normally realized by nouns.

This is simpler than it sounds. Consider these everyday words:

- 'The microorganism invades the body'

- 'The phagocyte moves into the area of invasion and attaches to the microorganism'

The first leaves the happening 'invades' as a happening: common wording. The second has nominalized 'invades' into 'invasion' and so transformed it into a thing: consolidated wording. Consolidateds have stronger ESD than commons. In this example, 'invasion' retains the meanings of 'invades' but additionally implies there are actors doing and/or subject to that action: 'invasion' suggests 'something invades something'. These need not be explicitly named, for they are packed into the consolidated word. Similarly in 'This period of invention is unmatched in history', the underlined consolidated renders a happening ('invents') as a thing ('invention'). It does not state who invents what but it does imply their existence and thereby signals potentially more relations to other meanings: stronger ESD. Consolidation may also render qualities as things, such as 'kind' as 'kindness', 'perfect' as 'perfection', etc. In 'Why is this longevity a problem for the atom?', the consolidated 'longevity' involves not only the quality 'long' (and its relations to other meanings) but also that which possesses the quality (and its relations to other meanings), suggesting stronger ESD ${ }^{13}$.

\subsubsection{Finest level of everydays}

As shown in Table 1, a further level of delicacy can be reached through distinctions within consolidated words and within common words.

First, we can distinguish within consolidated words between:

- specialist words or consolidateds located in texts dominated by technicals; and

- generalist words or consolidateds located in texts dominated by everydays.

13 Consolidation also facilitates a word's modification as if a thing (see section 4 on word-grouping) and its placement into further relations with other meanings, such as location within a taxonomy. 
This is to highlight the context within which consolidated words appear in the data being studied: the discourse of either specialized fields of practice (specialist) or everyday contexts (generalist). Specialist words are likely to exhibit stronger ESD than generalist words due to the kind of meanings they may be consolidating or packing inside themselves. While not depending for their meaning on a specialized field (i.e. not technicals), consolidateds surrounded by technicals are more likely to be packing up actors, things or entities that are technicals and thus more complex epistemic meanings. In so doing, they are likely to exhibit stronger ESD than consolidated-generalist words that are packing up everyday terms with less complex epistemic meanings. For example, the specialist word in 'The structure resulting from this fusion is called a phagolysosome' is likely to (and in this case did) involve consolidation of technicals and is being related to further technicals, whereas the generalist word in 'We have a bad connection on this phone' does not consolidate or relate to technicals.

Second, we can distinguish between two kinds of common words:

- nuanced words that offer relatively more differentiated meanings; and

- plain words that offer relatively general and less differentiated meanings.

Nuanced words exhibit stronger ESD because their greater differentiation implies relations to other possible forms. For example, consider these pairs of everyday-commons: devours/ eats, assassinate/kill, villa/house. In each pair the first word (nuanced) is more differentiated than the second word (plain): for example, a villa is one kind of house and can be distinguished from a cottage, bungalow, chalet, yurt, etc. Nuanceds thereby resonate out to more relations with other meanings, such as a tacit taxonomy of which the word is a member, suggesting potentially greater ESD ${ }^{14}$. This greater differentiation is likely to mean nuanced words are less frequently used in comparison to plain words which evoke fewer epistemic meanings in each instance of use but are highly flexible in terms of their potential sites of usage.

Assigning everyday-common words to these sub-subtypes may not be simple. The boundary is likely to be more fluid, shifting and subject to contestation than other categorical divisions. It is also likely to be the focus of relatively strong axiological-semantic density. Their relatively weak ESD leaves these words more sensitive to the context and more open to becoming markers of social categorization of actors. (For example, the English and Australian

14 In linguistic terms, plain words tend to be higher-order hyperonyms (or more generalized words) than the more specific hyponyms associated with nuanced words. 
co-authors of this paper could not agree on how to assign the words 'pretty' and 'beautiful'). However, despite this possible fluidity, we discovered many instances in the DISKS project data where the difference between nuanced and plain words had clear implications. For example, a secondary school teacher of biology repeatedly emphasized to students the importance of describing a phagocyte in their written work as 'engulfing and destroying' bacteria rather than 'eating and killing'. The relatively greater differentiation afforded by the nuanced versions was viewed by such teachers as significant for student achievement. Thus, even at the lowest levels of ESD, differences may have real effects.

\subsection{Proxy words}

Proxy words 'stand in' for other individual words. For example, in the following example, the word 'it' is used as a proxy three times for 'electron':

The electron can jump up to any point and it will fall back down in the end, and then if the energy supply is continuous it keeps getting thrown back up before it falls back down.

Proxy words may refer to all categories from the wording tool. In the above example, proxies refer back to a technical but they may also refer to everydays, such as in 'William needs to get a move on because he's falling behind'15. In terms of ESD strength, proxies are close but not identical to their referent words. For example, proxies of technicals are not themselves technicals; they may condense the same meanings in the discourse under consideration but they involve less direct resonance out to other meanings and less local differentiation. Put simply, proxies do not give the same sense of complexity to discourse as the words they reference and which themselves possess ESD. They are at one remove from the constellations of knowledge they evoke. In our illustrative analyses (section 5), we annotate proxies according to the category of their referents but with a superscript ' $P$ ' and we discuss them separately, in order to highlight their nature as condensers of complexity 'at one remove' and to signal their slightly weaker strength of ESD than other words of that type ${ }^{16}$. We should emphasize that here we are discussing proxies that refer to a single word. Words like 'it' or 'this' may also refer to longer stretches of discourse, which we discuss in Paper 2.

15 In linguistic terms, proxies tend to be reference items that refer to other words, though not all reference items are proxies - some are associated with aspects of sequencing (see Paper 2).

16 Although proxies may be a different linguistic category of word, we are concerned solely with ESD strengths, for which they are the same wording category as their referent item (with slightly weaker ESD). 


\section{Word-grouping}

The wording tool establishes basic strengths of ESD for English discourse. It provides a means of placing individual words on a continuum by assigning them to categories of relative strengths (see Figure 3, section 2.1). The word-grouping tool offers a means of fine-tuning those strengths for individual words. It shows how other words can boost a word's strength of ESD within a category by adding more meanings. The word-grouping tool is summarized in Table 3 and is applicable to all categories of words. It describes three types of word-grouping that strengthen ESD for words to different degrees: located, categorized and embedded.

\section{TABLE 3}

Word-grouping tool for epistemic-semantic density

\begin{tabular}{|l|l|}
\hline ESD & Type \\
\hline ESD $\uparrow \uparrow$ & embedded \\
\hline ESD $\uparrow$ & categorized \\
\hline ESD $\uparrow$ & located \\
\hline
\end{tabular}

Located modifications add meanings to a word by identifying a location in time or space, such as 'secretions on the skin' or 'diplomacy in the early $19^{\text {th }}$ century'. This enables greater differentiation by specifying the spatial or temporal domain over which the word refers but does not indicate a distinct taxonomy of types (such as other types of secretion or diplomacy).

Categorized modifications add meanings by specifying a distinct kind of the word, such as 'digestive enzyme', 'aesthetic trade', and 'source analysis'. This enables greater differentiation by making explicit both type (enzyme, trade, analysis) and subtype (digestive, aesthetic, source). It also suggests other subtypes and thus a potentially more complex taxonomy than 'located modifications', strengthening the ESD of the word more. (Additional subtypes could be specified, such as 'primary source analysis', further delineating potential taxonomic relations and thus strengthening ESD... until modifications become redundant).

Embedded modifications add meanings by showing explicitly that the word engages in an event or process through an embedded clause that follows the word, such as 'secretions that prevent microbial growth' and 'activities that could occur in a bath house'. These examples specify a particular type of secretion and a type of activity, respectively. They add mea- 
nings like categorized modifications but additionally specify a happening (event or process) and thereby the things involved in that happening. This implies more relations to meanings than sub-classifying alone, strengthening the ESD of the word more.

These three types can be combined; for example, 'other secretions on the skin that prevent microbial growth' specifies a location, a taxonomy, and a happening. The more modifications present for a word, the greater the increase in the strength of ESD (within the category established by the wording tool). Though hypothetically modifications could continue indefinitely, in practice it is likely to reach a limit as further modifications introduce redundant meanings. A final feature is that, just as individual words may be referred to by proxy (section 3.4), so can word-groupings. An example will be seen in section 5.1 where a teacher begins by quoting a word-group from an assessment task-'The influence of Greek and Egyptian cultures'-before asking 'What does that mean', where 'that' is a word-group proxy.

\section{Illustrative analyses of epistemic-semantic density}

The translation device is practical: the concepts outlined above offer a rough guide for allocating words to relative strengths of ESD. They are not a set of rules or 'cookie-cutter' grid. LCT analysis requires judgement in which broader knowledge of the data is invaluable, such as the context or whether specific words have been subjected to prior epistemological condensation. Moreover, not all the tools or levels of delicacy may be required. Here, for clarity and brevity, we shall illustrate the first two levels of delicacy (types and subtypes) of the wording tool and the word-grouping tool. It should be noted we are concerned only with the ESD demonstrated by wording and immediate word-grouping rather than attempting to capture the sum of meanings at play.

The key to annotation of the wording of examples is shown in Table 4: all technicals are in bold, with compacts plain bold and conglomerates in small capitals; words not in bold are everydays, with commons unadorned and CONSOLIDATEDS in small capitals. As mentioned in section 3.4., we annotate word-proxies according to their referents but with a superscript ' $P$ ', such as this ${ }^{\mathrm{P}}$ (excepting proxies for everyday-common words, the lowest ESD strength, as noting slight diminution of ESD offers little insight here). We discuss proxies after the main discussion of each example.

To facilitate reading, we shall annotate the excerpts for wording only and will discuss word-grouping afterwards, using the annotation shown in Table 5. The three forms are shown by square brackets [such as these] and a subscript number indicating a degree of strengthening of ESD: located as 1, categorized as 2, embedded as 3. (This is heuristic and not a scale). Note that word modifications can overlap: the number is after the final bracket for a modification. 


\section{TABLE 4}

Annotation for wording categories

\begin{tabular}{|c|c|c|}
\hline ESD & Type & Subtype \\
\hline+ & \multirow{2}{*}{ technical } & CONGLOMERATE \\
\hline & & compact \\
\hline & \multirow{2}{*}{ everyday } & CONSOLIDATED \\
\hline$\downarrow$ & & common \\
\hline
\end{tabular}

\section{TABLE 5}

Annotation for word-grouping categories

\begin{tabular}{|l|l|}
\hline Type & Annotation example \\
\hline embedded & {$[\text { secretions that prevent microbial growth }]_{3}$} \\
\hline categorized & {$[\text { lysosomal secretions }]_{2}$} \\
\hline located & {$[\text { secretions on the skin }]_{1}$} \\
\hline
\end{tabular}

Our examples are chosen to be contrastive for subject area (humanities/science), mode of discourse (spoken/written) and form of practice (pedagogy/research): an excerpt from a History lesson in Year 11 of secondary school in New South Wales, Australia; and the abstract of an article from a top-ranked scientific journal.

\subsection{Secondary school History example}

Our first example is a teacher explaining to a Year 11 History classroom how to answer a take-home assignment in which students are asked to evaluate 'the influence of Greek and Egyptian cultures in the Roman Empire'.

This is a little bit hard: "The InfLUENCE of Greek and Egyptian cultures." What does that mean? What would "the InfLUENCE of Greek and Egyptian cultures" mean, okay? No idea, right? What it means is, if we started to look at all the things in Pompeii and Herculaneum, what objects may be showing Greek design? Or Egyptian design? Or Greek mythology? Or Egyptian mythology? Or what building 
techniques like columns? Are there Greek columns? Do, you know, are the themes of their artwork reflecting it? So it's saying ...remember when we started, we said that Pompeii had originally been settled by Greeks? Okay? And if we look at where Italy is, it"'s not that far from Egypt at this time, umm, we've, we've had, umm ... Cleopatra has been killed by the time the volcano erupts, she ${ }^{p}$ and Mark Antony are dead and Egypt is part of the Roman Empire. So there would be massive amounts of TRADE going on, and umm, you know people visiting their diplomats you know or their, their, ambass...like their envoys, and things like that, all going back and forth across the countries. So, ideas. When you get TRADE in ideas - you wouldn't have heard this word before-we call it "aesthetic trade". Have you heard of it? Yeah.

Overall, the ESD of wording is at a lower level than our second example (section 5.2, below) and comprises mostly everydays, though several technicals punctuate the teacher's discussion. These technicals are compacts, the least strong technical words. This overall level of ESD reflects, we suggest, the discipline (History), stage of schooling (secondary) and mode (spoken).

The excerpt begins with the teacher highlighting the complex knowledge required by the assignment question by sandwiching a quotation from the question between 'This is a little bit hard' and 'No idea, right?'. The question itself represents relatively strong ESD: an everyday-consolidated ('INFLUENCE') is accompanied by three technicals ('Greek', 'Egyptian', 'culture'). The two names are technicals because they refer here to specific places and periods replete with specialized historical meanings. By this lesson in the unit, 'Greek' and 'Egyptian' have been related to specific customs, beliefs and practices ('culture') and located within both chronologies of history and taxonomic descriptions of social, economic and political systems. These technicals are combined with the technical 'culture' and everyday-consolidated 'INFLUENCE' in two categorized word-groupings that boost the strength of ESD further: "["The INFLUENCE of [Greek and Egyptian cultures $]_{2 .}$. $]_{2}$.

The teacher then asks the students to unpack some of the meanings condensed within these terms. After they fail to respond, the teacher begins doing so herself, mostly in everydays but with several technicals, such as 'Pompeii' and 'Herculaneum'. These words are compactelements, slightly less strong ESD than the compact-properties 'Greek' and 'Egyptian'. Furthermore, by using 'Pompeii' and 'Herculaneum' without any word-grouping modification, the ESD of her discourse here is weaker than the assignment question. Following this, the technicals 'Greek' and 'Egyptian' are repeated but rather than modifying other technicals, they are now modifying everydays, a weakening of ESD: '[Greek design $]_{2} \ldots[$ [Egyptian design] ... [Greek mythology $]_{2} \ldots$ [Egyptian mythology $]_{2} \ldots$ [Greek columns $]_{2}$ '. While 'design' or 'mythology' may 'sound' intuitively like technical words and may be technical in other settings, at this stage of secondary school History and in this class neither word has been situated within a developing constellation of historical knowledge and condensed with epistemological meanings. Such condensation may come later. However, the question for analysis is not whether 
a term might be technical somewhere else (such as later in the History curriculum or in other disciplines) but whether it is technical in the specific object of study under consideration. Here, these words possess weaker ESD.

After listing a series of technicals as possible issues to include in their work (up to 'Roman Empire'), the teacher explains how these might be related, addressing the key issue from the question of 'INFLUENCE'. At this point, she moves completely into everydays to describe processes that may relate the technicals she has enumerated:

So there would be massive amounts of TRADE going on, and umm, you know people visiting their diplomats you know or their, their, ambass... like their envoys, and things like that, all going back and forth across the see. So, ideas.

The teacher then repacks these ideas into the relatively strong ESD of 'aesthetic trade'. 'Trade' has now been condensed with epistemological meaning (we show how in Paper 2, section 4), transformed from an everyday into a technical and subclassified as 'aesthetic trade'. The technical 'trade' is additionally subjected to categorized modification by another technical, 'aesthetic': '[aesthetic trade $]_{2}$ '.

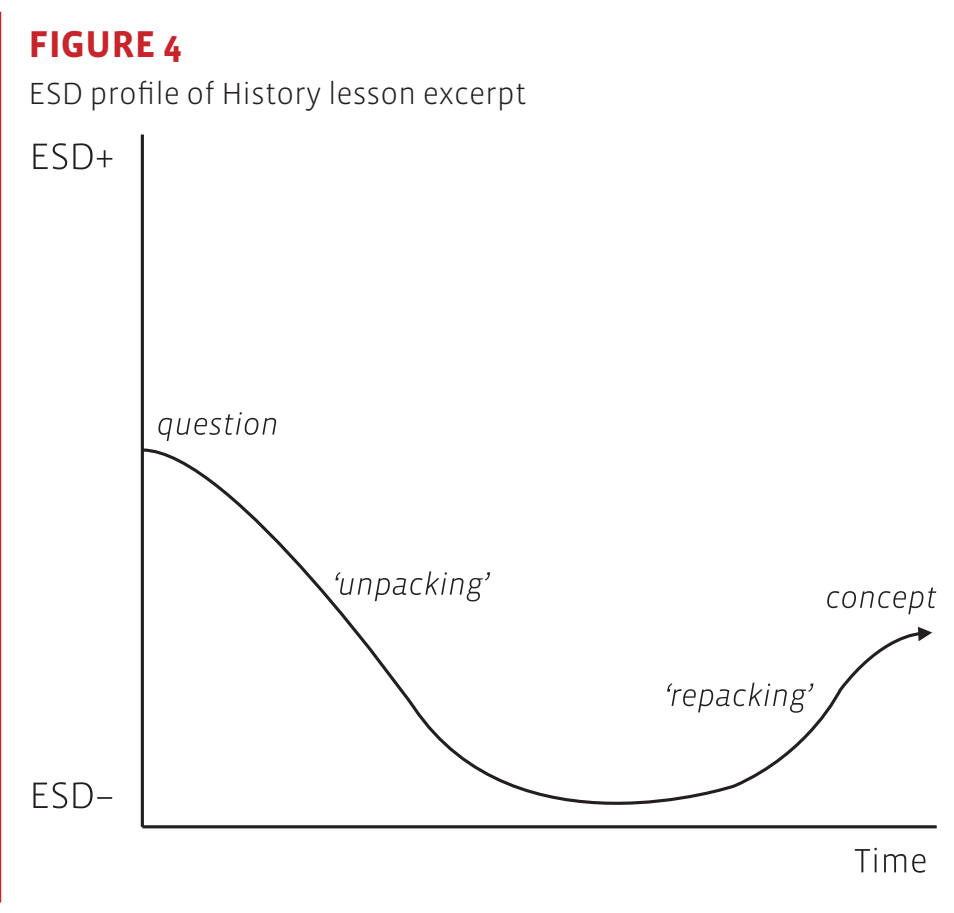

What, then, can enacting these tools tell us about the complexity of knowledge practices in this classroom excerpt? One way of synoptically displaying the ESD of wording over time is a profile (Maton, 2013, 2016b). Rather than tracing shifts between every single word, pro- 
files typically display a trend line that outlines the general location and pattern of change through the discourse. Figure 4 presents an ESD profile for the History classroom excerpt: beginning with relatively strong ESD wording, the discourse shifts into weaker ESD wording, before partially restrengthening ESD. This highlight how the teacher begins with the highly complex knowledge expressed by the assignment question, unpacks some of that complex knowledge using a series of simpler meanings, and then repacks those meanings into a concept ('aesthetic trade') that is more complex though less so than the initial question. In short, we can see how the teacher is employing wording that evokes different degrees of complexity in knowledge. She utilizes relatively simple technical words and everyday words to enumerate the many meanings being interrelated within the question and then brings some, though not all, these meanings together into a term of intermediate complexity. Of course, this is not the end of the knowledge being expressed. In this case, the teacher then went on to unpack and exemplify the concept of 'aesthetic trade', using again more everyday words, moving back down the profile.

\subsection{Scientific research example}

Our second example comprises the abstract for a scientific article published in Circulation, the top-ranked journal for cardiovascular disease (Suzuki et al., 2010: 1919):

Background - High-Density LIPOPROTEIN (HDL) protects the artery wall by removing cholesterol from lipid-laden macrophages. However, recent evidence suggests that hD might also inhibit atherogenesis by combating inflammation.

Method and Results - To identify potential anti-Inflammatory mechanisms we challenged MacropHAGES With LIPOPOLYSACCHARIDE, an inflammatory microbial ligand for Toll-like receptor 4. HDL inhibited the expression of 30\% (277 of 911) of the genes normally induced by LIPOPOLYSACCHARIDE, MICROARRAY analysis revealed. One of $\mathrm{ITS}^{\mathrm{p}}$ major targets was the type I interferon response pathway, a family of potent viral ImMUNOREGULAToRs controlled by Toll-like receptor 4 and the TRAM/TRIF signaling pathway. Unexpectedly, the ABILITY of HDL to inhibit gene expression was independent of MACROPHAGE cholesterol stores. IMMUNOFLUORESCENT studies suggested that HDL promoted tRAM TRANSLOCATION TO INTRACELLULAR compartments, which impaired subsequent signaling by Toll-like receptor 4 and TRIF. To examine the potential in vivo relevance of the pathway, we used mice deficient in APOLIPOPROTEIN A-I, the major protein of HDL. After infection with Salmonella typhimurium, a Gramnegative bacterium that expresses LIPOPOLYSACCHARIDE, APOLIPOPROTEIN A-I-deficient mice had 6-fold higher plasma levels of interferon- $\boldsymbol{\beta}$, a key regulator of the type-I interferon response, than did wild-type mice.

Conclusions - HDL inhibits a subset of LIPOPOLYSACCHARIDE-stimulated MACROPHAGE genes that regulate the type I interferon response, and its ${ }^{\mathrm{p}}$ ACTION is independent of sterol metabolism. These findings raise the POSSIBILITY that REGULATION Of MACROPHAGE genes by HDL might link innate immunity and CARDIOPROTECTION. 
Overall, the abstract embodies a far stronger ESD than our first example. This is unsurprising given it is written, research and science rather than spoken, pedagogy and humanities. This is manifested by the proportion of technicals to everydays; indeed, a high number of these technicals are conglomerates, the strongest ESD wording. Moreover, there are numerous instances of technicals modifying other technicals and thereby further strengthening their ESD through word-grouping.

Such strengthening is exemplified by the start of the 'Conclusion':

HDL inhibits [[a subset of [LIPOPOLYSACCHARIDE-[stimulated [MACROPHAGE genes $\left.\left.\left.]_{2}\right]_{2}\right]_{2}\right]_{2}$ that regulate the $\left.\left.[\text { type I [interferon response }]_{2}\right]_{2}\right]_{3}$

This begins with the conglomerate 'HDL' (an acronym of 'High-Density Lipoprotein') being said to inhibit 'a subset of LIPOPOLYSACCHARIDE-stimulated MACROPHAGE genes that regulate the type I interferon response', a word-group comprising eight technicals, including two conglomerates. This word-group is an example of an embedded modification, the strongest form, with 'genes' followed by the modification 'that regulate the type I interferon response'. Within this wordgroup, there are six other modifications, all categorized, such as the four modifications in: '[a subset of [LIPOPOLYSACCHARIDE -[stimulated[MACROPHAGE genes $\left.\left.\left.]_{2}\right]_{2}\right]_{2}\right]_{2}$ '. The compounding of wordgrouping upon word-grouping involving technicals modifying technicals greatly strengthens the ESD of the text. Put simply, a lot of already complex meanings are being related together in numerous ways to generate even greater complexity. The lack of explication of their meanings in the text assumes the target reader grasps a considerable degree of highly complex knowledge. In other words, the abstract suggests considerable epistemological condensation has already occurred elsewhere. It is thus 'insider' discourse, understanding of which requires a prolonged training in the constellations of knowledge being evoked.

The extremely strong ESD is unrelenting through the excerpt. Each sentence comprises on average 10 technicals (between 3 and 19), with a high proportion being conglomerates. Moreover, all except one sentence involves numerous word-groupings in which technicals modify technicals. When everydays are used, they are often consolidateds, the stronger ESD form of everyday words ('ACTION', 'POSSIBILITY', 'REGULATION') or nuanced forms of commons ('evidence', 'independent', 'inhibits'). In short, the text consistently utilizes stronger ESD words and word-groupings wherever possible. Yet, there remains room for the discourse to explore even more complexity at the wording level. Exploring the third level of delicacy of the wording tool reveals that almost all the conglomerates in this text are cong/omerate-elements (e.g. 'LipopoLYSACCHARIDE', 'APOLIPOPROTEIN') rather than the highest ESD form of conglomerate-properties (e.g. 'AtHEROGENESIS' and 'CARDioprotection').

In comparison to the history example, there are very few proxies at either wording or word-grouping level. This is likely a function of the type of text: a scientific abstract must co- 
ver a large degree of meaning in a small number of words, leaving little scope to describe and discuss words and word-groups over several sentences. Thus each sentence offers a high degree of ESD before moving on, limiting the need for proxies to track meanings across the text.

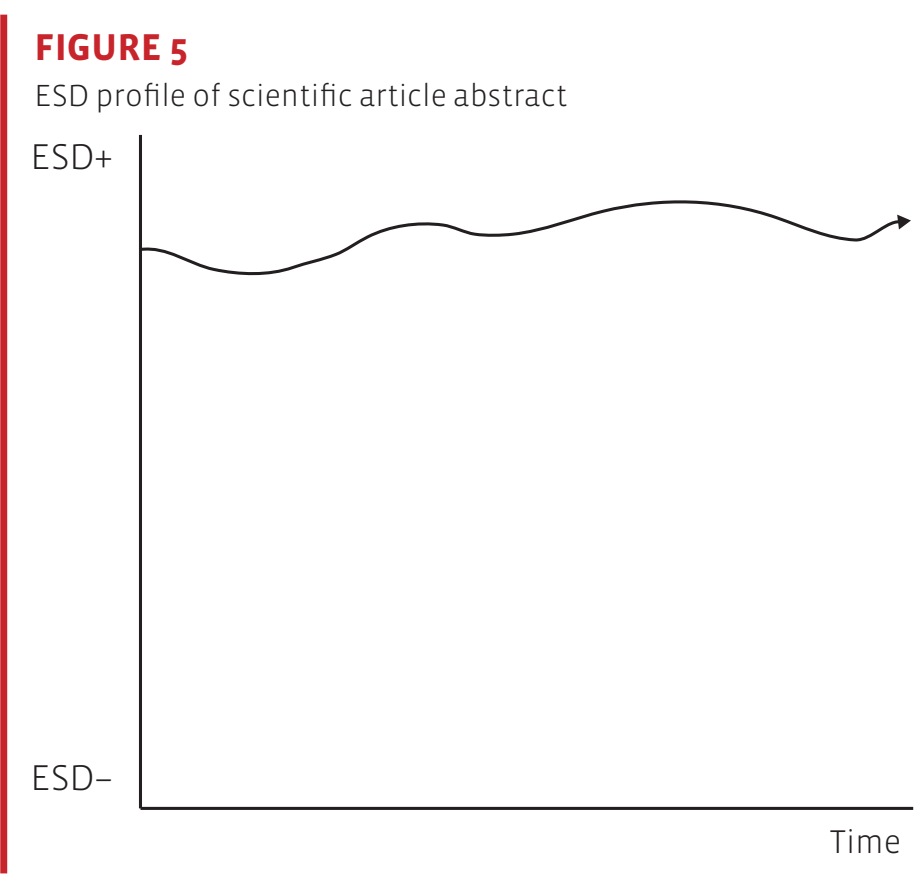

In terms of the ESD profile, as shown in Figure 5, drawing a trend line reveals what is termed a high flatline (Maton, 2013) ${ }^{17}$. The profile is set at a much higher level than the History classroom excerpt (Figure 4), reflecting the more complex knowledge expressed and, unlike the classroom example, there is relatively little movement here. The discourse remains within a symbolic domain of complex epistemological constellations of knowledge.

\section{Conclusion}

The complexity of knowledge practices is a key issue for understanding the bases of achievement in educational contexts, how these 'rules of the game' vary across subject areas, through stages of education and between research, curriculum and pedagogy, and how they can be taught more equitably. This paper introduced the concept of 'semantic density' from LCT as a means of theorizing the complexity of knowledge practices. Our principal focus has been

17 This is a flatline of ESD only; analysis of semantic gravity might reveal a different profile. 
to outline tools that enable the epistemic form of this flexible concept to be enacted in the study of English discourse. In this paper we defined typologies that help 'read' the wording used by actors for signs of epistemic complexity of knowledge. One does not need these tools to explore ESD in practices. For example, Maton (2013) analysed the History classroom excerpt and outlined a similar semantic profile prior to the development of these concepts. However, by providing a more explicit and systematic basis for exploring the ESD revealed by discourse, the tools help make the gaze which generated that analysis more widely accessible, replicable and open to scrutiny (cf. Maton, 2016a). Similarly, we emphasized that enacting the tools requires judgement, as exemplified by the classroom analysis that designated 'culture' as a technical and 'mythology' as an everyday based on our knowledge of previous lessons. Nonetheless, the methodological basis of such judgements can also be made explicit. For example, distinguishing technicals from everydays can draw on artefacts viewed as authoritative by actors in the field (dictionaries, glossaries, content pages, curriculum, content points, etc.), and cross-checking by 'insiders' can ensure reliability and validity of the analysis.

The proof of the concept, however, is in the explanation. Typically we anticipate the tools will be enacted to help explain a precise problem, such as the basis of achievement in student assignments or the nature of cumulative knowledge-building in pedagogic practices. Here, space limited us to two brief analyses of contrastive examples in order to illustrate how the tools can be enacted. Nonetheless, they suggest how the concepts can account for one organizing principle shaping different kinds of knowledge practices expressed in the discourse used by actors. They also raise questions for further study, including why the History classroom traces a wave pattern and the science abstract is a flatline, what drives movements of ESD up and down in the former, and whether the latter might involve movements of a different kind. More generally, the tools allow us to trace the changing degree of complexity expressed by the wording of actors but not how meanings are being condensed into words over time. That is, profiles portray successive states of the ESD of words rather than changes in the ESD of those words; they reveal the changing nature of knowledge but now how that knowledge is being built into constellations. It is to this issue of condensation and knowledge-building that we now turn, in the second of these two linked papers on complexity.

\section{Bibliographic references}

Bernstein, B., 2000: Pedagogy, Symbolic Control and Identity: Theory, research, critique, London: Rowman \& Littlefield.

BLACKIE, M., 2014: "Creating semantic waves: Using Legitimation Code Theory as a tool to aid the teaching of chemistry", Chemistry Education Research and Practice 15, 462-9. 
Calado, S., I. P. Neves \& A. M. Morais, 2013: “Conceptual demand of science curricula: A study at the middle school level”, Pedagogies 8(3), 255-77.

Chen, R. T-H., K. Maton \& S. Bennett, 2011: "Absenting discipline: Constructivist approaches in online learning" in F. Christie and K. MAton (eds.): Disciplinarity: Functional linguistic and sociological perspectives, London: Continuum, 129-50.

Clarence, S., 2014: Enabling Cumulative Knowledge-building Through Teaching: A Legitimation Code Theory analysis of pedagogic practice in law and political science. Unpublished PhD thesis, Rhodes University, South Africa [available at http://www.legitimationcodetheory.com].

Georgiou, H., K. Maton \& M. Sharma, 2014: "Recovering knowledge for physics education research: Exploring the 'Icarus effect' in student work", Canadian Journal of Science, Mathematics, and Technology Education 14(3), 252-68.

GlenN, E., 2015: From Clashing to Matching: Examining the legitimation codes that underpin shifting views about climate change. Unpublished PhD thesis, Institute for Sustainable Futures, University of Technology Sydney.

Macnaught, L., K. Maton, J. R. Martin \& E. Matruglio, 2013: "Jointly constructing semantic waves: Implications for teacher training", Linguistics and Education 24(1), 50-63

MARTIN, J. R., 2013a: "Embedded literacy: Knowledge as meaning" Linguistics and Education $24(1), 23-37$.

Martin, J. R. \& E. Matruglio, 2013b: "Revisiting mode: Context in/dependency in Ancient History classroom discourse", Studies in Functional Linguistics and Discourse Analysis 5, 72-95.

Martin, J. R., 2013C: Interviews With M. A. K. Halliday, London: Bloomsbury.

Martin, J. R., K. Maton \& Matruglio, E., 2010: "Historical cosmologies: Epistemology and axiology in Australian secondary school history discourse", Revista Signos 43(74), 433-63.

MAton, K., 2009: "Cumulative and segmented learning: Exploring the role of curriculum structures in knowledge-building", British Journal of Sociology of Education 30(1), 43-57.

Maton, K., 2011: "Theories and things: The semantics of disciplinarity" in F. Christie \& K. Maton (eds.): Disciplinarity: Functional linguistic and sociological perspectives, London: Continuum, 62-84.

Maton, K., 2013: “Making semantic waves: A key to cumulative knowledge-building”, Linguistics and Education 24(1), 8-22. 
Maton, K., 2014a: "Building powerful knowledge: The significance of semantic waves" in B. BARRETT \& E. RATA (eds.): Knowledge and the Future of the Curriculum, London: Palgrave Macmillan, 181-197.

Maton, K., 2014b: Knowledge and Knowers: Towards a realist sociology of education, London: Routledge.

Maton, K., 2016a: "Legitimation Code Theory: Building knowledge about knowledge-building" in K. Maton, S. Hood \& S. Shay (eds.): Knowledge-building: Educational studies in Legitimation Code Theory, London: Routledge, 1-24.

Maton, K., 2016b: "Starting points: Resources and architectural glossary" in K. Maton, S. Hood \& S. SHAY (eds.): Knowledge-building: Educational studies in Legitimation Code Theory, London: Routledge, 233-43.

Maton, K. \& R. T-H. Chen, 2016: "LCT in qualitative research: Creating a translation device for studying constructivist pedagogy" in K. Maton, S. Hood \& S. ShaY (eds.): Knowledge-building: Educational studies in Legitimation Code Theory, London: Routledge, 27-48.

Maton, K. \& Y. J. Doran, 2017: "SFL and code theory" in T. BARTLETt \& G. O'Grady (eds.): The Routledge Systemic Functional Linguistic Handbook, London: Routledge.

Maton, K., J. R. Martin \& E. Matruglio, 2016a: "LCT and systemic functional linguistics: Enacting complementary theories for explanatory power" in K. Maton, S. Hood \& S. SHAy (eds.): Knowledgebuilding: Educational studies in Legitimation Code Theory, London: Routledge, 93-113.

Maton, K., S. Hood \& S. Shay (eds.), 2016b: Knowledge-building: Educational studies in Legitimation Code Theory, London: Routledge.

Matruglio, E., K. Maton \& J. R. Martin, 2013: "Time travel: The role of temporality in enabling semantic waves in secondary school teaching", Linguistics and Education 24(1), 38-49.

Morais, A., I. Neves \& D. Pires, 2004: "The what and the how of teaching and learning" in J. Muller, B. Davies \& A. Morals (eds.): Reading Bernstein, Researching Bernstein, London: Routledge Falmer, 75-90.

Suzukı, M. et al., 2010: "High-density lipoprotein suppresses the type I interferon response, a family of potent antiviral immunoregulators, in macrophages challenged with lipopolysaccharide", Circulation 122, 1919-27. 